University of South Carolina

Scholar Commons

10-19-1987

\title{
Normal-Metal Aharonov-Bohm Effect in the Presence of a Transverse Electric Field
}

\author{
S. Washburn \\ H. Schmid \\ D. Kern
}

Richard A. Webb

University of South Carolina - Columbia, webbra@mailbox.sc.edu

Follow this and additional works at: https://scholarcommons.sc.edu/phys_facpub

Part of the Physics Commons

\section{Publication Info}

Published in Physical Review Letters, Volume 59, Issue 16, 1987, pages 1791-1794.

Washburn, S., Schmid, H., Kern, D., and Webb, R.A. (1987). Normal-Metal Aharonov-Bohm Effect in the Presence of a Transverse Electric Field. Physical Review Letters, 59(16), 1791-1794. doi: 10.1103/ PhysRevLett.59.1791

(C) 1987 The American Physical Society.

This Article is brought to you by the Physics and Astronomy, Department of at Scholar Commons. It has been accepted for inclusion in Faculty Publications by an authorized administrator of Scholar Commons. For more information, please contact digres@mailbox.sc.edu. 


\title{
PHYSICAL REVIEW LETTERS
}

\begin{tabular}{lcc}
\hline \hline Volume 59 & 19 OCTOBER 1987 & Number 16 \\
\hline \hline
\end{tabular}

\section{Normal-Metal Aharonov-Bohm Effect in the Presence of a Transverse Electric Field}

\author{
S. Washburn, H. Schmid, D. Kern, and R. A. Webb \\ IBM Thomas J. Watson Research Center, Yorktown Heights, New York 10598 \\ (Received 22 June 1987)
}

\begin{abstract}
The effects of transverse electric fields on the conductance fluctuations in an Sb loop have been studied. We show that the electric field can be used to tune the position (or phase) of Aharonov-Bohm oscillations as well as to alter the aperiodic conductance fluctuation patterns. We discuss two mechanisms which might cause the observed dependence of the fluctuation pattern on transverse electric field. The first is the electrostatic Aharonov-Bohm effect, and the second is the spatial shifting of the electron trajectories by the electric field.
\end{abstract}

PACS numbers: $05.40 .+\mathrm{j}, 03.65 . \mathrm{Bz}, 72.15 . \mathrm{Gd}$

As a result of their extreme sensitivity to the phase coherence of the wave function, magnetoresistance fluctuations ${ }^{1}$ seen in small devices, which are caused by Aharonov-Bohm ${ }^{2}$ effects among the various electron trajectories in the sample, particularly the oscillations in metal loops, ${ }^{3,4}$ are proving to be a powerful tool in the study of electron transport. The large, "universal" values $^{5}$ of the fluctuations seen in metallic samples at low temperatures ${ }^{6}$ have been used to study the phasecoherence length $L_{\phi}$ of the electrons, ${ }^{7}$ the correlations of the individual levels in the conductance band, ${ }^{8,9}$ correlations between potential fluctuations in disordered materi$\mathrm{al},{ }^{10}$ the effects of paramagnetic impurities on electron phase coherence, ${ }^{11}$ and the effects of ensemble averaging. ${ }^{12}$ In brief, the Aharonov-Bohm effect is the modulation of the phase of the electron wave functions by electromagnetic potentials. Usually, attempts to observe Aharonov-Bohm effects ${ }^{4,13}$ rely on the interaction of the vector potential $\mathbf{A}$ with the electron wave function through the line integral $\int \mathbf{A} \cdot d \mathbf{s}$ where $s$ is the path of the electron. A beam of electrons is split between two paths and then recombined. Any magnetic flux threaded between the two paths shifts the relative phase of the wave functions in the two paths. Because of this interaction with the field, the magnetoresistance of a metal loop oscillates with period $\Delta H=(h / e) / a$, where $a$ is the area enclosed by the loop. In a disordered medium the carrier trajectories are a random tangle of paths. This randomness adds random factors to the resistance fluctuations which, in turn, change the smooth periodic dependence to a random speckle pattern which contains local periodicity. ${ }^{14}$

The phase of the wave function can also be changed by the application of an electric field. This electric field contributes the fourth term in the four-vector product $A_{\mu}(d x)^{\mu}$, and its effects have been studied previously in electron-beam interference experiments performed in vacuum. ${ }^{15}$ This term contains the scalar potential $V$ associated with transverse electric fields and the time. It causes a phase shift in the wave function of $\Delta \phi$ $=\int e V d t / \hbar$. The usual Ansatz is to suppose that a scalar potential is in contact with the electron wave function for some distance along its trajectory and to calculate the time interval $d t$ from the path length and the Fermi velocity $v_{\mathrm{F}}$. For a disordered material with no inelastic scattering, this velocity would be replaced by the drift velocity. In most practical realizations of metal samples, however, the drift velocity is so low that the time interval is set by the phase-coherence time $\tau_{\phi}$. It was recently proposed that the application of a electric field perpendicular both to the current path and to the magnetic field would induce such an Aharonov-Bohm oscillation. ${ }^{16}$ The prediction from a two-channel model of a loop in the ballistic regime was that if the transverse electric field was present between the electron paths, then it could be used to modulate the Aharanov-Bohm oscillations.

We have designed a simple experimental arrangement to search for electric-field effects in metal loops. Figure 


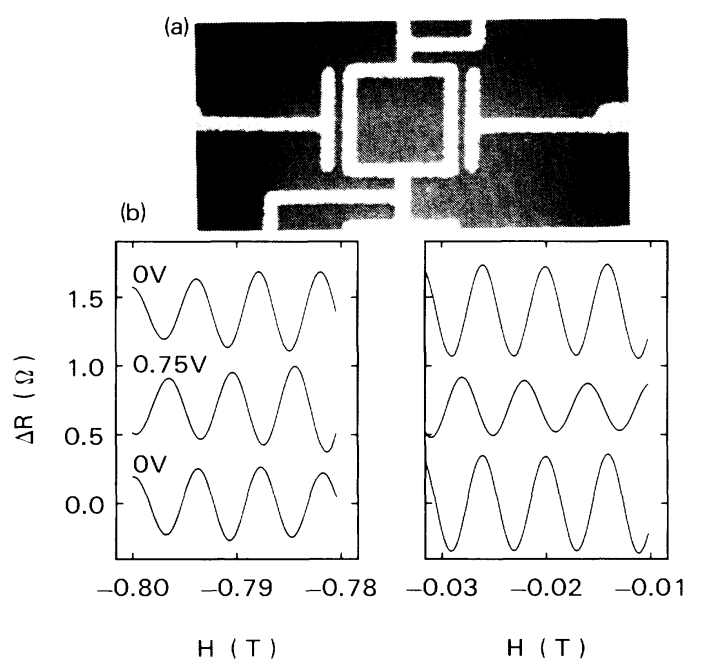

FIG. 1. (a) Photograph of the Sb loop used in this experiment. The capacitor probes are about $0.8 \mu \mathrm{m}$ long and about $0.16 \mu \mathrm{m}$ from the arms of the loop (center to center). (b) The effect on the Aharonov-Bohm oscillations of a voltage applied across the capacitor in two ranges of magnetic field. (The aperiodic fluctuations have been filtered for clarity of display.)

1 (a) is a photograph of the device. The loop is $0.82 \mu \mathrm{m}$ on a side, and the capacitive probes (left and right) are $0.16 \mu \mathrm{m}$ from the arms of the loop. All components of the device were formed in a single processing step on a Si substrate with conventional electron-beam liftoff technology. The device comprises Sb $(99.9999 \%$ pure) wires which are approximately square in cross section being $d \simeq 0.075 \mu \mathrm{m}$ thick and wide, and the resistance between the voltage probes is $136 \Omega$. (The material $\mathrm{Sb}$ was chosen because it yields large phase-coherence lengths and large resistivity.) We calculate that the capacitance between one of the probes and the loop is of the order of $5 \times 10^{-17} \mathrm{~F}$; the presence of surface charge on the $\mathrm{Si}$ could increase this coupling by 2 or 3 orders of magnitude. We emphasize here that it is not obvious that an externally applied electric field will produce a potential difference between the left and right arms of the ring. In fact, for a perfect conductor, it is forbidden by classical electrostatics. The applied electric field, however, will certainly affect the charge distribution on some of the surfaces of the loop.

The data in Fig. 1(b) clearly demonstrate that the voltage on the capacitor probes can be used to tune the positions of the $h / e$ oscillations in the loop. We plot the $h / e$ resistance oscillations for two selected magnetic field ranges at $40 \mathrm{mK}$. The top traces are for zero voltage difference across the capacitor probes. The middle traces demonstrate that after the application of $V=0.75$ $\mathrm{V}$ across the capacitor, the phase of the $h / e$ oscillations is changed by nearly $\pi$, and the bottom traces show that when the voltage difference is returned to zero the $h / e$
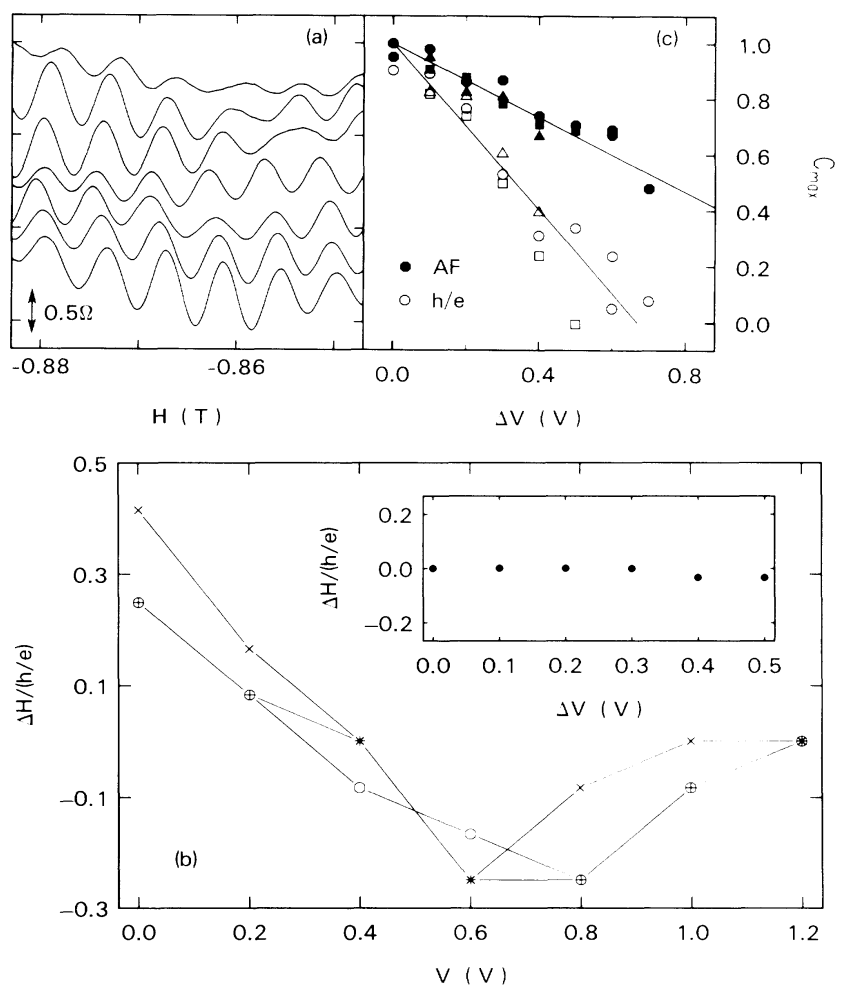

FIG. 2. (a) Magnetoresistance curves recorded at several values of the applied dc electric field illustrating the shift in the oscillations and changes in the random components in the conductance fluctuations. The applied voltage is lowest for the top trace and increments by $0.2 \mathrm{~V}$ on each subsequent trace. (b) The positions of the peaks in (a) as a function of applied voltage. The various symbols refer to different peaks in the magnetoresistance. Inset: The position of the maximum in $C(A, B)$ calculated after the magnetoresistance curves ( $A$ and $B$ ) have been filtered to remove the aperiodic fluctuations. (c) The maximum amplitude of the cross-correlation function $C(A, B)$ as a function of the difference in applied electric field for the aperiodic fluctuations (solid symbols) and for the $h / e$ oscillations (open symbols).

oscillations return to their original phase. Notice that in the two different magnetic field ranges, the oscillations shift by slightly different amounts.

Upon performing a sequence of such experiments for various voltages between the capacitor probes, one obtains data which, at least locally, are consistent with the prediction that the electric field will simply add an extra phase shift to the oscillations. An altogether typical sequence of magnetoresistance traces at different voltages is shown in Fig. 2(a) to illustrate this behavior. The traces include aperiodic fluctuations (AF) having an average amplitude $\Delta G$ [AF] $=0.4 e^{2} / h$ and periodic oscillations of amplitude $\Delta G[h / e]=0.2 e^{2} / h$. The phase of the oscillations shifts as the voltage on the capacitor probes changes. Moreover, the oscillations in a given region move approximately as a unit; i.e., the entire mag- 
netoresistance curve appears to slide along the field axis as a unit. This is illustrated in Fig. 2(b), where the relative positions (referred to the positions at $V=1.2 \mathrm{~V}$ ) of a few of the local maxima in the magnetoresistance are plotted as a function of $V$. The positions of the oscillations shift smoothly and more or less uniformly with electric field. This would appear to be evidence that the transverse electric field adds to the phase of the electrons in the same way that the magnetic vector potential adds to them. We emphasize here that the voltage scale in Fig. 2(b) is very large compared with what we estimate for the electrostatic Aharonov-Bohm effect. The voltage required to phase shift the wave function by $2 \pi$ is $V_{2 \pi}=2 \pi \hbar / e \tau_{\phi}=40 \mu \mathrm{V}$, which is smaller by 5 orders of magnitude than the voltage difference on the capacitor plates.

In contrast to the case of electrons propagating freely in a vacuum where the electric Aharonov-Bohm effect has been seen previously, ${ }^{15}$ the conductance fluctuations, which we measure here, contain several terms which depend critically on the mixture of electron trajectories. The formula for the conductance $g=G /\left(e^{2} / h\right)$ contains fluctuation terms such as

$$
\Delta g=g_{0}(H, V)+g_{1}(H, V) \cos \left\{[2 \pi \Phi(H, V) /(h / e)]+\alpha_{1}(H, V)\right\}+\cdots .
$$

The random factors $g_{0}, g_{1}$, and $\alpha_{1}$ are present in the conductance formula because of the scrambling of the wave functions caused by the impurity scattering. Not only does the electric field add to the usual Aharonov-Bohm phase $\Phi(H, V)$, it also causes random fluctuations in $g_{0}$, $g_{1}$, and $\alpha_{1}$.

To illustrate this complexity, we refer again to the sequence of magnetoresistances in Fig. 2(a). Not only do the oscillations shift, but the envelope function $g_{1}$ and the random term $g_{0}$ also change shape. In addition, the phase of the oscillations $\alpha_{1}$ changes randomly at the nodes in $g_{1}$. It is not surprising that these terms are random functions of electric field since the electric field should have all the same effects as the magnetic field. As a result of this random dependence on the electric field, analysis of the type displayed in Fig. 2(b) cannot be carried to arbitrary voltage scales because the oscillations which are being mapped simply disappear as nodes in $g_{1}$ move through the pattern. The random dependence of $g_{1}$ on $V$ can be illustrated by the calculation of the cross-correlation function $C(A, B)$ for the magnetoresistances (the magnetoresistance trace $A$ being recorded at a given voltage between the capacitor plates and $B$ recorded at another) and plotting the maximum amplitude $C_{\max }$ of $C(A, B)$ as a function of the difference in applied voltage. Using a digital filter, we can independently study the aperiodic fluctuations and the periodic $h / e$ oscillations because the characteristic frequency scales are well separated in our experiment. These results are displayed in Fig. 2(c) for the $h / e$ and aperiodic conductance fluctuations separately. For the aperiodic fluctuations, $C_{\max }$ decays as the voltage difference increases $\left(C_{\max }=0.5\right.$ at $\left.\Delta V=0.7 \mathrm{~V}\right)$ which indicates that the dc electric field also changes $g_{0}$ randomly. It has, therefore, the same effect on this term that the magnetic field has: It causes aperiodic fluctuations. A similar effect is observed for the $h / e$ oscillations but $C(A, B)$ decays more quickly with voltage, $C_{\max }=0.5$ at $\Delta V \simeq 0.35 \mathrm{~V}$. If the only effect of the electric field had been to shift the magnetoresistance oscillations along the magnetic field axis, then instead of decaying monotonically, the cross-correlation function would have oscillated about zero with the period $\Delta V=V_{2 \pi}$. This difference be- tween the half-width for aperiodic fluctuations and the periodic oscillations is somewhat perplexing. We expect all of the factors in Eq. (1) to have about the same scale for their dependences of electric field because the net phase shift in the wave function is determined by the time of "contact" between the wave function and the scalar potential. We note that there is a similar difference between the characteristic magnetic-flux scale for $h / e$ oscillations and aperiodic fluctuations with the former being $h / e$ and the latter $\simeq 2 h / e$. The rolloff of the correlation does not depend on how the voltage is applied. If $+V / 2$ (referenced to the circuit which measures the loop resistance) is applied to the left-hand probe and $-V / 2$ applied to the right-hand probe, the resulting voltage correlation graph is the same as when all of the voltage is applied to one probe, and the other probe is held at ground (the potential of the measurement circuit). If the positions of the cross-correlation maxima are plotted as a function of the applied dc electric field, then [see the inset in Fig. 2(b)] on average, the shift of the oscillations is seen to be zero. This is the result of the random dependence of $\alpha_{1}$ on the applied voltage. Since this term changes randomly with electric field, the shift of the oscillations averaged over a wide range of magnetic field (in this case a range of $2 \mathrm{~T}$ or $300 \mathrm{~h} / \mathrm{e}$ through the loop) is washed out. In other words, the slight difference between the two shifts in Fig. 1(b) is indicative of the randomness inherent in the speckle pattern. Data in other ranges of magnetic field yield shifts which are distributed randomly between 0 and $2 \pi$.

It is very tempting to attribute all of the above observations to the quantum-mechanical effects of the scalar potential on the wave function. There are some difficulties, however, with this interpretation. The electrostatic potential could not extend into the metal for distances larger than the screening length $\left(l_{s} \simeq 2 \AA\right)$, over which distance it decays exponentially. The phase shift in the electron wave functions from the electrostatic Aharonov-Bohm effect would then be accumulated only when the electrons move within a screening length of the edge of the arms of the loop. In order to obtain a simple estimate of the reduction of $V$ by screening, we make the 
following argument: The fraction of time spent "in contact" with the scalar potential is just the ratio of the unscreened amount of wire to the total amount of wire. Based on the assumption that there is no discontinuity in the dielectric constant at the Sb surface, the effective voltage across the loop $V_{\mathrm{eff}} \simeq\left(l_{s} / x\right)\left(l_{s} / d\right) V=5 \times 10^{-6}$ $\mathrm{V}$, where $x$ is the separation (edge to edge) between the capacitor and the loop. This brings the measured voltage scale $V_{2 \pi} \simeq 2 \mathrm{~V}$ to within 1 order of magnitude of the expected $40 \mu \mathrm{V}$. We suspect that the dielectric discontinuity at the Sb surface and the high dielectric constant of the $\mathrm{Si}$ substrate will both tend to reduce $V_{\text {eff }}$ further. The electron wave functions penetrate into the $\mathrm{Si}$ substrate $^{17}$ on distances of the same order $\left(l_{\text {pen }} \simeq 1 \AA\right)$. If there is no inversion charge screening the electric field at the $\mathrm{Si}$ surface, then the electrons in the Sb might "contact" the electrostatic potential in the substrate. The reduction of the applied voltage is also difficult to estimate in this case because the extent of the wave-function tail into the substrate and the thickness and height of the Schottky barriers are difficult to calculate. We suspect that the reduction will again be many orders of magnitude. Alternatively, if the capacitor probes leak current into the loop through the various Schottky barriers and a surface charge layer, then a potential gradient can exist. Our measurements suggest that this gradient is much less than a nanovolt, and therefore, is unimportant here.

The classical electrostatic force on the electron must also be present. It is, after all, the conduction electrons which screen the electric fields in the loop. Although the force on the electrons in the loop is small, it is still large enough to move many of the electron trajectories. Since, in the spirit of the theory which discusses the effects of moving a single impurity, ${ }^{18}$ it is only necessary to move few of the classical trajectories a distance of order of the Fermi wavelength $\left(\lambda_{\mathrm{F}}=10 \AA\right)$ in order to change the pattern of fluctuations, it is probable that the classical force is affecting the measurements. It is obvious that spatially shifting the classical trajectories could give rise to the results in Fig. 2(c) where the half-width of the cross-correlation function is interpreted as the amount of voltage required to move the trajectories a distance $\lambda_{\mathrm{F}}$. A gradual shift of the classical trajectories might lead to the results in Fig. 2(b) by gradually scrambling the speckle pattern. Since the net effect of scrambling the paths is random, we expect that the average shift in the conductance fluctuations (measured globally by a correlation function) will be zero which is consistent with the inset in Fig. 2(b).

To conclude, we have demonstrated experimentally that a transverse electric field can be used to tune the conductance fluctuations in a metal loop. The effects of electric field are in all cases similar to that of the perpendicular magnetic field, as one expects from the Aharonov-Bohm mechanism, but because of the disorder in the device, it is impossible to say whether it is this mechanism or simply the spatial rearrangement of the carrier trajectories which causes the observed effects. There are classical forces on the electrons in the loop, and these forces must play a role in the voltage dependence.

We gratefully acknowledge technical assistance from M. Berger, P. Chang, B. Grossman, C. van Haesendonck, C. Umbach, and R. Laibowitz. We are indebted to R. Landauer for useful discussions.

${ }^{1}$ R. A. Webb, S. Washburn, C. P. Umbach, and R. B. Laibowitz, in Localization, Interaction, and Transport Phenomena in Impure Metals, edited by G. Bergmann, Y. Bruynseraede, and B. Kramer (Springer-Verlag, Heidelberg, 1985); C. P. Umbach, S. Washburn, R. B. Laibowitz, and R. A. Webb, Phys. Rev. B 30, 4048 (1984).

${ }^{2}$ Y. Aharonov and D. Bohm, Phys. Rev. 115, 485 (1959).

${ }^{3}$ D. Yu. Sharvin and Yu. V. Sharvin, Pis'ma Zh. Eksp. Teor. Fiz. 34, 285 (1981) [JETP Lett. 34, 272 (1981)].

${ }^{4}$ R. A. Webb, S. Washburn, C. P. Umbach, and R. B. Laibowitz, Phys. Rev. Lett. 54, 2696 (1985); V. Chandrasekhar, M. J. Rooks, S. Wind, and D. E. Prober, Phys. Rev. Lett. 55, 1610 (1985); S. Datta et al., Phys. Rev. Lett. 55, 2344 (1985).

${ }^{5}$ P. A. Lee, A. D. Stone, and H. Fukuyama, Phys. Rev. B 35 , 1039 (1987).

${ }^{6}$ S. Washburn and R. A. Webb, Adv. Phys. 35, 375 (1986).

${ }^{7}$ F. P. Milliken, S. Washburn, C. P. Umbach, R. B. Laibowitz, and R. A. Webb, Phys. Rev. B 36, 4465 (1987).

${ }^{8}$ J. C. Licini, D. J. Bishop, M. A.Kastner, and J. Melngailis, Phys. Rev. Lett. 55, 2987 (1985).

${ }^{9}$ S. Washburn, C. P. Umbach, R. B. Laibowitz, and R. A. Webb, Phys. Rev. B 32, 4789 (1985).

${ }^{10}$ A. Benoit, C. P. Umbach, R. B. Laibowitz, and R. A Webb, Phys. Rev. Lett. 58, 2343 (1987); W. J. Skocpol et al., Phys. Rev. Lett. 58, 2347 (1987).

${ }^{11}$ A. D. Benoit et al., unpublished.

${ }^{12}$ C. P. Umbach, C. van Haesendonck, R. B. Laibowitz, S. Washburn, and R. A. Webb, Phys. Rev. Lett. 56, 386 (1986); W. J. Skocpol et al., Phys. Rev. Lett. 56, 2865 (1986).

${ }^{13}$ A. Tonomura et al., Phys. Rev. Lett. 48, 1443 (1982).

${ }^{14}$ Y. Gefen, Y. Imry, and M. Ya. Azbel, Phys. Rev. Lett. 52, 129 (1984); M. Büttiker, Y. Imry, R. Landauer, and S. Pinhas, Phys. Rev. B 31, 6207 (1985).

${ }^{15} \mathrm{H}$. Schmid, in Proceedings of the Eighth European Congress on Electron Microscopy, Budapest, Hungary, 1984, edited by A. Csanady, P. Rohlich, and D. Szabo (Programme Committee of the Congress, Budapest, 1984), p. 285.

${ }^{16}$ S. Datta, M. R. Melloch, S. Bandyopadhyay, and M. S. Lundstrom, Appl. Phys. Lett. 48, 487 (1986).

${ }^{17}$ N. D. Lang, in Solid State Physics, edited by H. Ehrenreich, F. Seitz, and D. Turnbull (Academic, New York, 1973), Vol. 28, p. 225, et seq.

${ }^{18}$ B. L. Al'tshuler and B. Z. Spivak, Pis'ma Zh. Eksp. Teor. Fiz. 42, 363 (1985) [JETP Lett. 42, 447 (1985)]; S. Feng, P. A. Lee, and A. D. Stone, Phys. Rev. Lett. 56, 1960 (1986). 


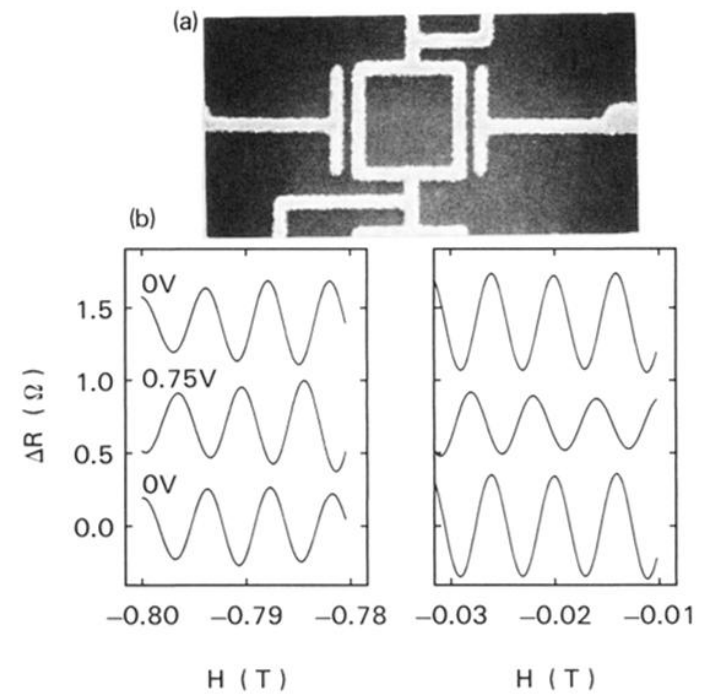

FIG. 1. (a) Photograph of the Sb loop used in this experiment. The capacitor probes are about $0.8 \mu \mathrm{m}$ long and about $0.16 \mu \mathrm{m}$ from the arms of the loop (center to center). (b) The effect on the Aharonov-Bohm oscillations of a voltage applied across the capacitor in two ranges of magnetic field. (The aperiodic fluctuations have been filtered for clarity of display.) 